\title{
Analysis of fixturing quality for a variable number of fixturing points and friction values
}

\author{
F. Penalba, J. Rosell and R. Suárez
}

\begin{abstract}
This paper copes with the automatic determination of fixturing points on 2D and 3D free-form objects, for any number of fixturing points and a variable friction coefficient at the contacts. A software tool that implements the searching algorithm, and that is also able to analyze the quality of any given fixturing, is presented. An analysis on 2D and 3D objects has been performed with this tool, that allows to determine how many points are necessary and which coefficient of friction is required in each case in order to fix the object with a given quality. The tool has been released as open software.
\end{abstract}

\section{INTRODUCTION}

A key point in a manufacturing process is the proper fixturing of objects when they are going to be processed in any way, or some particular actions must be done on them. There is a number of well known examples, like for instance polishing, drilling, or just performing an assembly of subparts to form a more complete product, among several others. In this situations there is always at least one part that must keep its position despite the application of external forces on it, in order to successfully perform the desired action. There are several works dealing with the problem of object fixturing, considering different particular conditions and/or constraints, including a number of works presented in the field of grasping and manipulation, which has several points in common with the problem of fixturing.

Relevant concepts in this field are the form-closure property (the position of the fixtures/fingers ensures the object immobility) and force-closure property (the forces applied by the fixtures/fingers ensure the object immobility) [1]. The force-closure constraint is more frequently required in grasping, since the movement of the object makes its own weight to act as an external perturbation, while the formclosure constraint is more frequently required in fixturing, where the object usually lies in a stable position while no operation in being performed on it. Some relevant works dealing with grasping and fixturing of objects based on this property are given below in Section 2 .

In this work we present a tool to decide which is the most convenient way to restrict the position of an abject assuring a desired minimum quality in terms of the forces that the object can resist without loosing the position. We have extended a previous work [2] and made an implementation that allows the search and analysis of fixturing points on the object. The idea is to visualize the quality of potential object fixturings

This work was partially supported by the Spanish Government through the projects DPI2007-63665 and DPI2008-02448.

The authors are with the Institute of Industrial and Control Engineering (IOC), Technical University of Catalonia (UPC), Barcelona, Spain. jan.rosell@upc.edu. under different conditions, like the number of contact points and the friction coefficient at those points, and use this information to decide how to secure the object. The approach is valid for $2 \mathrm{D}$ and $3 \mathrm{D}$ objects.

The paper is organized as follows. After this introduction, Section 2 presents the approach used to find force and form closure fixturings and to evaluate their quality. Section 3 describes the implemented tool (software) developed to search and analyze object fixturings. Section 4 shows some application examples in order to illustrated the approach. Finally Section 5 summarizes the work, presents some conclusions and discusses future work and potential improvements.

\section{Fixturing Search and Evaluation}

\section{A. Related background}

How to constrain an object in a desired position depends on a number of factors, being the most relevant: the dimension of the object, i.e. $2 \mathrm{D}$ or $3 \mathrm{D}$, the object shape, i.e. polyhedral or non-polyhedral, the type of contact between the fixtures (or fingers) and the object, i.e. frictionless, frictional or soft contact, and the number of contacts (which for 3D objects must be equal or larger than 2 when soft contacts are considered, equal or larger than 4 for frictional contacts, and equal or larger than 7 for frictionless contacts). See [3] for a review of these factors. These different cases are addressed in several relevant works, considering, for instance, 2D polygonal objects [4], 2D non-polygonal objects [5], 2D discrete objects [6], 3D polyhedral objects [7], [8], 3D non-polygonal objects [9], [10], and 3D discrete objects [11]-[13].

Arbitrary shaped objects are frequently modeled with a finite (but large) number of points, either using clouds of points as samples of the object surface or any type of mesh. These models are quite convenient when the object boundary is obtained using range sensors, or some vision systems based on structured light [14], [15], and they can also easily be obtained from any other representation. In this work we consider the boundary of a 3D object to be described by a triangular mesh, while a $2 \mathrm{D}$ object boundary is directly described by a finite sequence of discrete points.

\section{B. Quality measures}

Several different quality measures were presented in the literature to evaluate the performance of a given fixture or grasp. See [16] for a survey on grasp quality measures. The quality measures that take into account the object properties (shape, size, weight), friction constraints and form and force closure conditions to quantify the grasp quality, can be classified into three subgroups. The first two groups do not 
consider limitations in the magnitudes of the forces applied at the contact points, one group considers only algebraic properties of the grasp matrix (for instance the value of its minimum singular value that indicates how far is the grasp from a singular configuration [17]), and the other group considers geometric relations in the grasp (for instance the shape [18] and the area [19] of the polygon defined by a three contact point fixture). The third group considers limitations in the magnitudes of the forces applied to constrain the object, thus being more realistic for practical applications. The quality measures used in this work belong to this third group, although constraints derived from the indeterminate friction forces in a quasi-static analysis [20] are not included. Given the forces that can be applied on the object at the contact points, the produced wrenches on the object are known, and they are used to compute the following quality measures:

a) The radius of the largest hypersphere centered at the origin of the wrench space and fully contained in the Convex Hull of the wrenches that can be applied on the object at the contact points [21], [22], which indicates the maximum wrench that the constrained object can resist independently of the wrench direction. This measure depends on the reference point used to compute the torques.

b) The volumen of the the Convex Hull of the wrenches that can be applied on the object at the contact points [23], which gives an idea of the amount of wrenches that the object can resist, and is constant independently of the reference system used to compute torques.

\section{Implemented approach}

The approach used to compute a force closure grasp and to evaluate its quality is a generalization of the work presented in [2], which deals with the case of seven frictionless contact points for 3D discrete objects. That work is extended to allow the application to 2D and 3D objects, and using any number of contacts, either frictionless or frictional. Some other added features are described below.

The main algorithm, valid for 2D and 3D objects and any number of frictionless or frictional contacts, is as follows:

Step 1: Generate an initial set $G p$ of $m$ fixturing points and evaluate its quality.

Step 2: Select another point $\boldsymbol{p}_{j}$ on the object surface.

Step 3: Select a particular point $\boldsymbol{p}_{i} \in G p$

Step 4: Evaluate the resultant quality when $\boldsymbol{p}_{i}$ is replaced by $\boldsymbol{p}_{j}$ in $G p$.

Step 5: If the quality grows then update $G p$ replacing $\boldsymbol{p}_{i}$ by $\boldsymbol{p}_{j}$.

Step 6: While a finishing condition is not satisfied go to Step 2.

The generation of the initial set $G p$ of $m$ fixturing points in Step 1, as well as the other points in Step 2, is done using a sampling procedure that tries to pick points uniformly distributed over the object surface. Random and deterministic sampling algorithms were used for this purpose. The first point of $G p$ is randomly selected, and the remaining $m-1$ points of $G p$ and the rest of the points in Step 2 are either

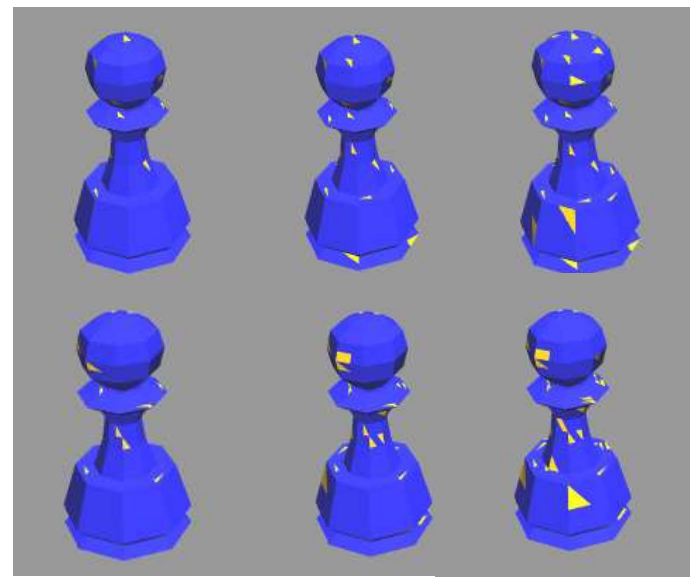

Fig. 1. Illustration of the deterministic sampling (top) and random sampling (bottom) on the object surface of a pawn model for 20,30 and 40 samples from left to right, respectively.

randomly selected or selected maximizing the distance to the already selected points, in this latter way the object surface is better uniformly sampled (Fig. 1). The distance between points can be measured in different ways as, for instance, using a Euclidean distance between any two points or using the number of points in the mesh between them (details about the implemented solutions are given in next section). This generation of samples is iteratively repeated until any termination condition is satisfied.

The evaluation, in Steps 1 and 4, of the fixturing quality produced by the set of contact points $G p$ is computed using any of the two criteria presented in Subsection II-B.

The selection of a particular point $\boldsymbol{p}_{i} \in G p$ in Step 3 is done such that, once a new point $\boldsymbol{p}_{j}$ is selected on the object surface, the direction of the wrench $\boldsymbol{w}_{i}$ produced by the normal force applied on $\boldsymbol{p}_{i}$ is the closest to the direction of the wrench $\boldsymbol{w}_{j}$ produced by the normal force applied on $\boldsymbol{p}_{j}$. This criterion tends to minimize the change in the directions of the potential wrenches applied on the object and facilitates the convergence of the algorithm.

Step 5 is straightforward, and, finally, the finishing condition in Step 6 can be any of the followings:

- A given desired minimum quality is obtained.

- A given number of steps without improving the quality were performed.

- A given number of points on the object surface were visited.

- All the points on the object surface were visited.

\section{A TOOL TO ANALYZE THE FIXTURES}

There exists a powerful tool, Graspit! [24], that is focused on grasp planning, providing procedures to find the best grasp of a given object with a given mechanical hand. In our work we are interested in analyzing some properties of fixturings only from the object point of view, in particular the relation between the number of points, the friction coefficients at the contacts and the fixturing quality that can be obtained. For this reason, a tool called Grasp Analysis Tool (GAT) has been implemented to find 

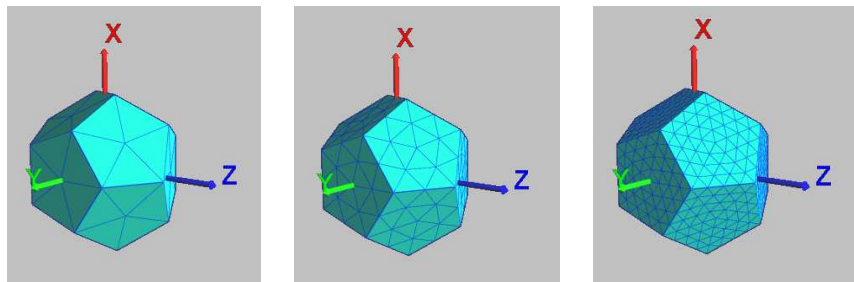

Fig. 2. Model refinement process on a dodecahedron. From left to right, model composed of 60, 240 and 960 triangles, respectively.

fixturing or grasping points for a given object following the algorithm presented in the previous section. The tool can also be used to evaluate the quality of any set of given fixturing or grasping points. It has basically been implemented with an analysis aim and, thus, the user can define many parameters related to the object models used, the type of fixtures, the quality measures or some parameters of the search algorithm. They are detailed in the following subsections. The software package can be downloaded from http://iocnet.upc.edu/usuaris/JanRosell/GAT/GAT.html.

\section{A. Object models}

The Grasp Analysis Tool works for free-form objects in two and three dimensions: 2D objects are defined as a closed line described by a set of points; 3D objects are defined as a closed volume described with a triangular mesh. The segments defined by two consecutive points in 2D, or the triangles in $3 \mathrm{D}$, are called elements. Their geometric center define the candidate fixturing or grasping points. Therefore, the search algorithm obtains better results with models composed of many uniform elements.

The tool has an option that allows to refine the models by subdividing all their segments or triangles as illustrated in Fig. 2. Also, a parameter, $\lambda$, is defined to scale the objects. Assuming unitary forces at the contact points, the parameter $\lambda$ is used to scale the torques, i.e. $w_{i}=\left(f_{i}, \lambda \tau_{i}\right)$.

\section{B. Type of fixtures}

Fixtures vary as a function of the number of fixturing points and as a function of the force directions that can be exerted at them, which is determined by the friction coefficient at the contacts.

In the Grasp Analysis Tool, the effect of friction is introduced by defining the friction coefficient $\mu$, considered equal at all the contact points. For the $3 \mathrm{D}$ case, the friction cone is approximated by a polyhedral convex cone with eight sides. The frictionless option is also available.

For the frictionless case the number of points ranges from 4 and 7 for the 2D and 3D cases, respectively, up to the number of elements of the model. When friction is considered, the minimum number of points is set to 3 and 4 , respectively.

Fig. 3 shows the interface devoted to the configuration of these parameters.

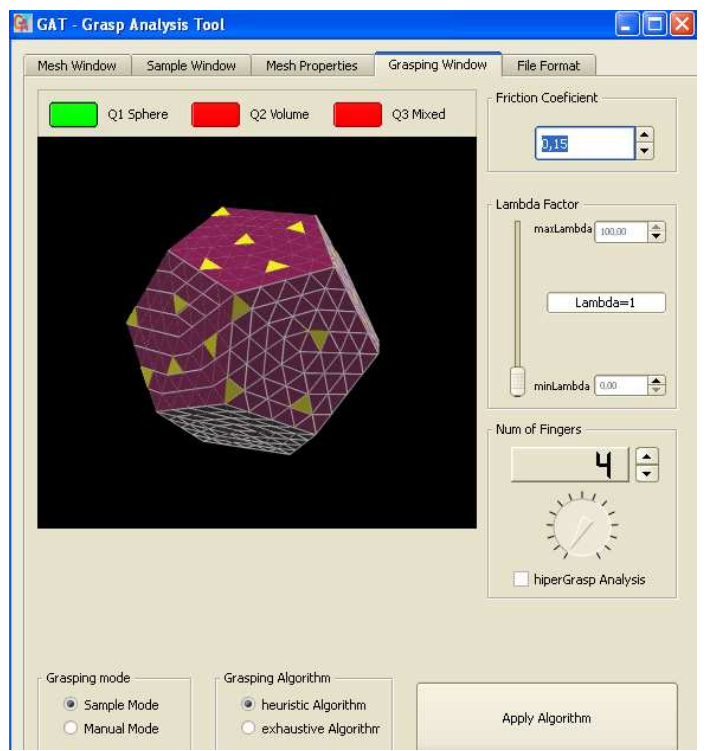

Fig. 3. Interface to determine the type of fixture: number of points and friction coefficient.

\section{Quality measures}

The two used quality measures have been described in Section II-B and a combination of them has also been implemented as a third option. They are labelled as:

- $Q_{1}$ : The radius of the maximum hypersphere.

- $Q_{2}$ : The volume of the Convex Hull.

\section{Parameters of the searching algorithm}

The tool allows the searching algorithm to be run with different sampling strategies and with different distance measures:

a) Sampling strategies: Deterministic or random sampling strategies can be chosen, as illustrated in Fig. 4, being the number of points to be sampled also variable. The manual selection of the candidate points is also possible.

For objects with few elements considered as potential fixturing points, the algorithm can be run in an exhaustive way, i.e. all the combinations are tested and the one with the best quality is chosen.

b) Distance measure: Distance between two elements is computed by the propagation of the distance between neighbor elements, thus, several alternatives exist. First, for 3D models two type of neighborhood can be defined: a) Standard neighborhood: two triangles are considered neighbors if they share an edge; b) Extended neighborhood: two triangles are considered neighbors if they share at least one vertex (Fig. 5). Second, the distance between neighboring triangles can be defined in two ways: a) Discrete distance: neighboring triangles are at a distance one; b) Euclidean distance: the distance between neighboring triangles is computed as the Euclidean distance between their centers.

\section{ANALYSIS OF FIXTURING QUALITY: EXAMPLES}

This section uses the GAT tool to analyze how the fixturing quality depends on the number of fixturing points and on the friction coefficient. The examples are based on 


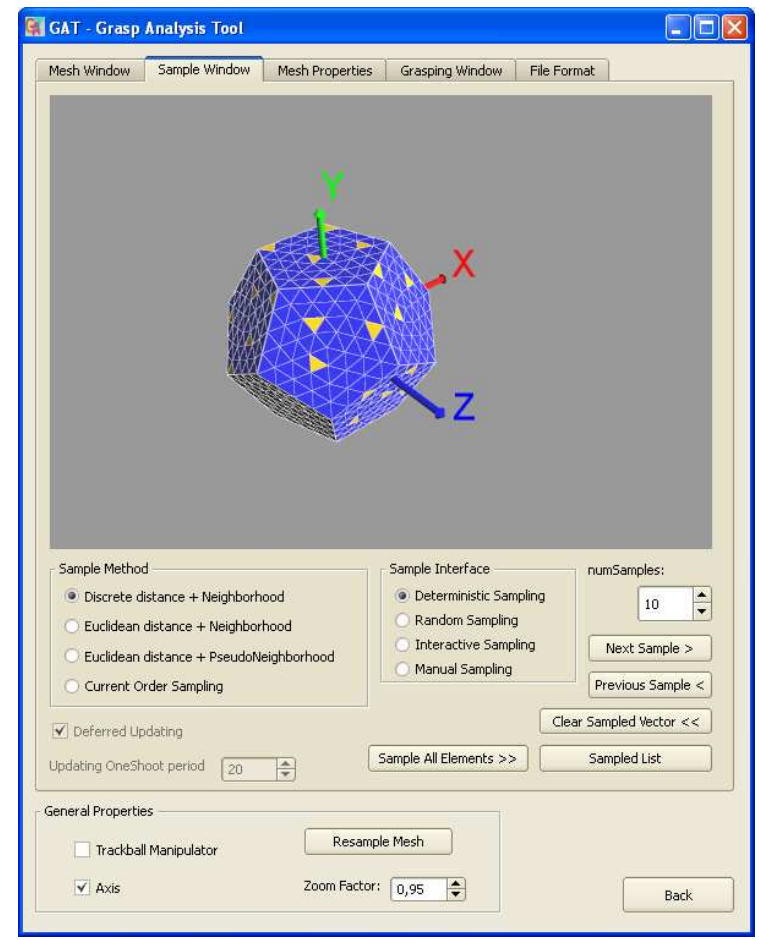

Fig. 4. Interface to select the desired type of sampling.

the application of the searching algorithm on 2D and 3D models with the following parameters (see Subsections III-C and III-D):

- Quality measure: $Q_{1}$.

- Sampling sequence: Deterministic.

- Distance measure: Euclidian distance combined with extended neighborhood.

- Friction values: $0.01,0.05,0.10,0.15,0.20$ and 0.25 .

- Number of fixturing points: from 3 to 11 for the 2D case and from 4 to 11 for the 3D case.

Since the search algorithm is heuristic, no optimal result can be guaranteed. Therefore, each example has been run three times starting each time with a different element on the object surface. The chosen starting elements are: a) the closest element to the geometric center of the object; $b$ ) the furthest element to the geometric center of the object; c) a randomly selected element.

For each example, the quality obtained by the algorithm for each friction value and for each number of fixturing points is graphically reported. This quality is the maximum obtained by running the algorithm from the three considered starting points.

\section{A. $2 D$ examples}

Two 2D examples have been considered: a rectangle and an ellipse. In both cases, the algorithm has been run until all the points on the object perimeter have been visited.

The rectangle has an aspect ratio $3 \times 1$. The initial model, composed of eight uniform elements, has been refined up to 256 elements, resulting each element with a size lower than the $0.4 \%$ of the total perimeter. The ellipse has an aspect ratio $2 \times 1$. The model is composed of 400 non-uniform
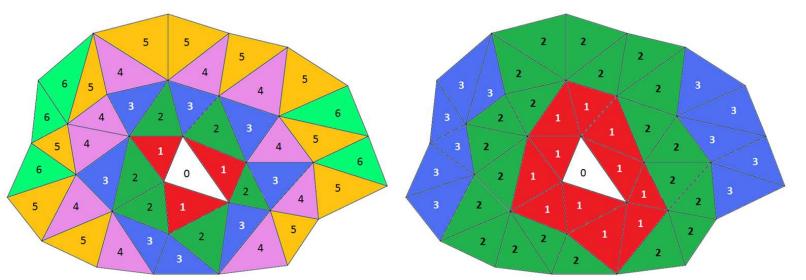

Fig. 5. Discrete distance from a given triangle (white) to the other triangles in the mesh, computed using standard neighborhood (left) and extended neighborhood (right).

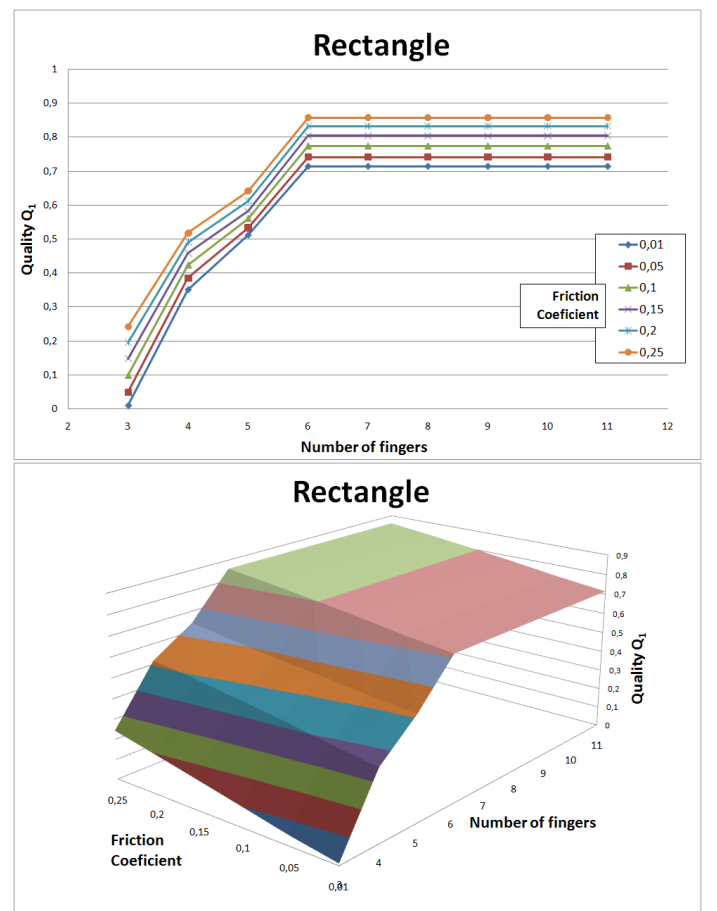

Fig. 6. Experiment results with the Rectangle: (top) quality $Q_{1}$ vs. number of fixturing points for different friction coefficients; (bottom) quality $Q_{1}$ vs. both number of fixturing points and friction coefficients.

elements, being their size lower than the $0.35 \%$ of the total perimeter.

Figures 6 and 7 show the results. As expected, it can be seen that the quality increases with the number of fixturing points and with the friction coefficient. In both cases this increase is not relevant for more than 6 fixturing points, being even for the ellipse not much relevant from 4 fixturing points. The increase in the friction coefficient is, on the other hand, always relevant, irrespective of the number of fixturing points used.

\section{B. $3 D$ examples}

Three 3D examples have been considered (Fig. 8): two regular polyhedra (a tetrahedron and a dodecahedron), and an irregular object (a pawn). For the tetrahedron, the algorithm has been run until all the points on the object surface have been visited. For the other two examples the number of visited points has been limited due to the computational time needed for the complete exploration, and of the very slow increase in the quality that is obtained once a representative number of points have been visited, as illustrated in [2]. 


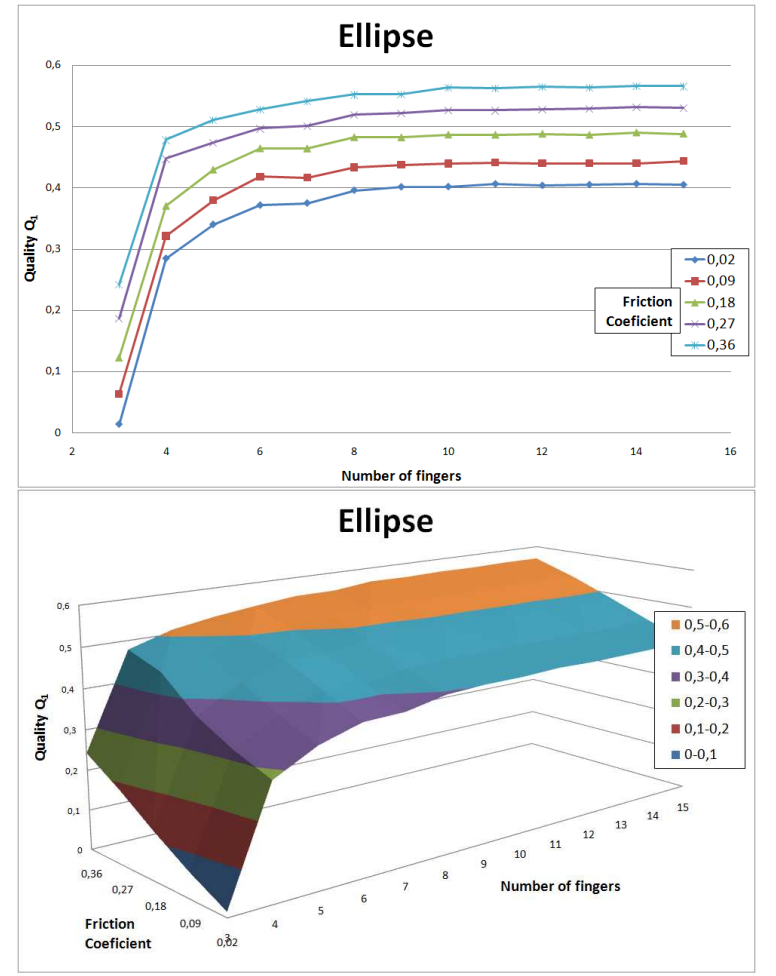

Fig. 7. Experiment results with the Ellipse: (top) quality $Q_{1}$ vs. number of fixturing points for different friction coefficients; (bottom) quality $Q_{1}$ vs. both number of fixturing points and friction coefficients.
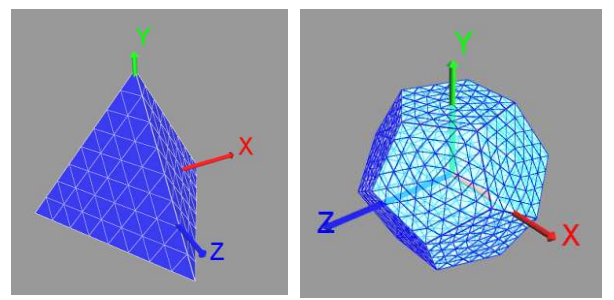

Fig. 8. 3D examples.

The initial model of the tetrahedron, composed of only four triangles, has been refined up to 256 , resulting each triangle with an area lower than the $0.4 \%$ of the total area. The initial model of the dodecahedron, composed of 60 triangles, has been refined up to 960 , resulting each triangle with an area lower than the $0.15 \%$ of the total area. The number of sampled triangles was set to 200 . The initial model of the pawn, composed of 304 triangles, has been refined up to 1216 , resulting each triangle with an area lower than the $0.4 \%$ of the total area. The number of sampled triangles was set to 300 .

Figures 9,10 and 11 show the results for the tetrahedron, the dodecahedron and the pawn, respectively. As in the $2 \mathrm{D}$ examples, the quality increases with the number of fixturing points and with the friction coefficient.

For the tetrahedron, the grasping quality presents a staircase shape with respect to the number of fixturing points, i.e. there are flat regions between 4 and 6 points and between 8 and 10. Therefore it makes nonsense to use 5 or 6 points instead of 4 since the quality is nearly the same, and for the
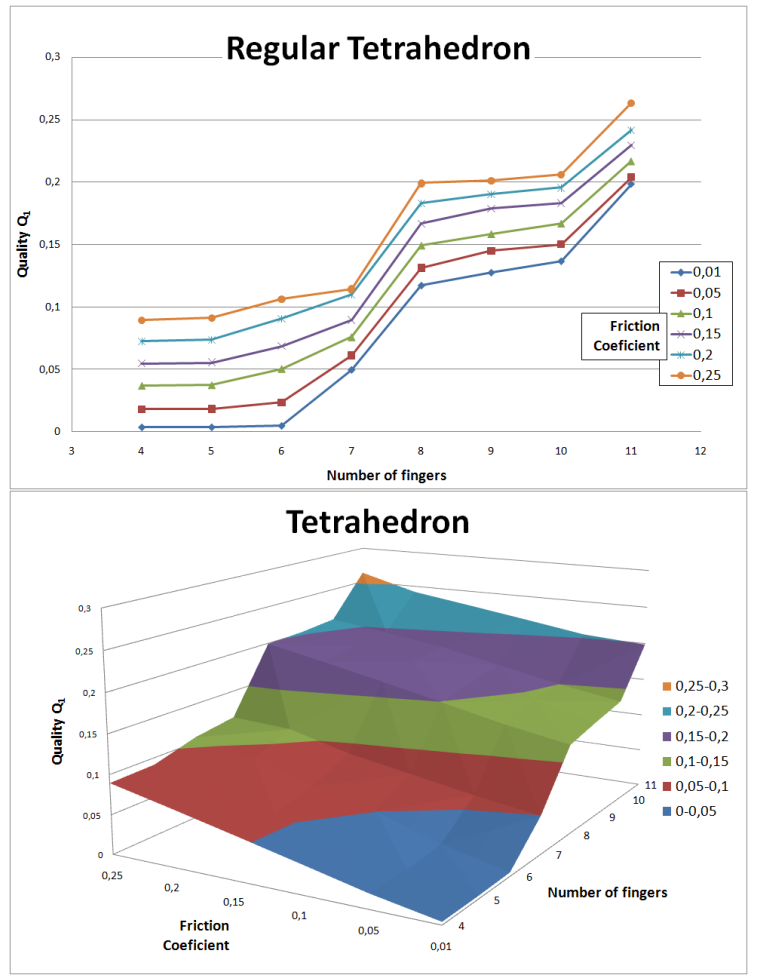

Fig. 9. Experiment results with the Tetrahedron.

same reason it makes nonsense to use 9 or 10 points instead of 8 .

For the dodecahedron there is a very important increase of quality when incrementing the number of fixturing points from 5 to 7 , which motivates the use of a number of fixturing points equal to or larger than 7 .

For the pawn it can be observed that for low friction coefficients there is a considerable quality step between 6 and 7 fingers, therefore in this case a reasonable number of fixturing points is 7 or more. On the other hand, for high friction coefficients the behavior of the quality is almost linear with the number of fixturing points, which suggests the use of as many points as possible but without a minimum number required.

In all cases, there is a linear increase as a function of the friction coefficient, irrespective of the number of fixturing points, although this linearity is not so clear for the case of the pawn. Then, the quality is always directly increased by an increase in the friction coefficient.

\section{CONCLUSIONS}

This paper has analyzed how the fixturing quality of $2 \mathrm{D}$ or 3D free-form objects depends on the number of fixturing points and on the friction coeficient at those points. Fixturing points are found by an heuristic algorithm previously proposed by the authors that has been generalized to both $2 \mathrm{D}$ and $3 \mathrm{D}$ objects, to friction or frictionless contacts, and to a variable number of fixturing points. A software tool has been implemented to automate this analysis. The results on several objects allow to select in each case the minimum number of fixturing points and friction coefficient required 

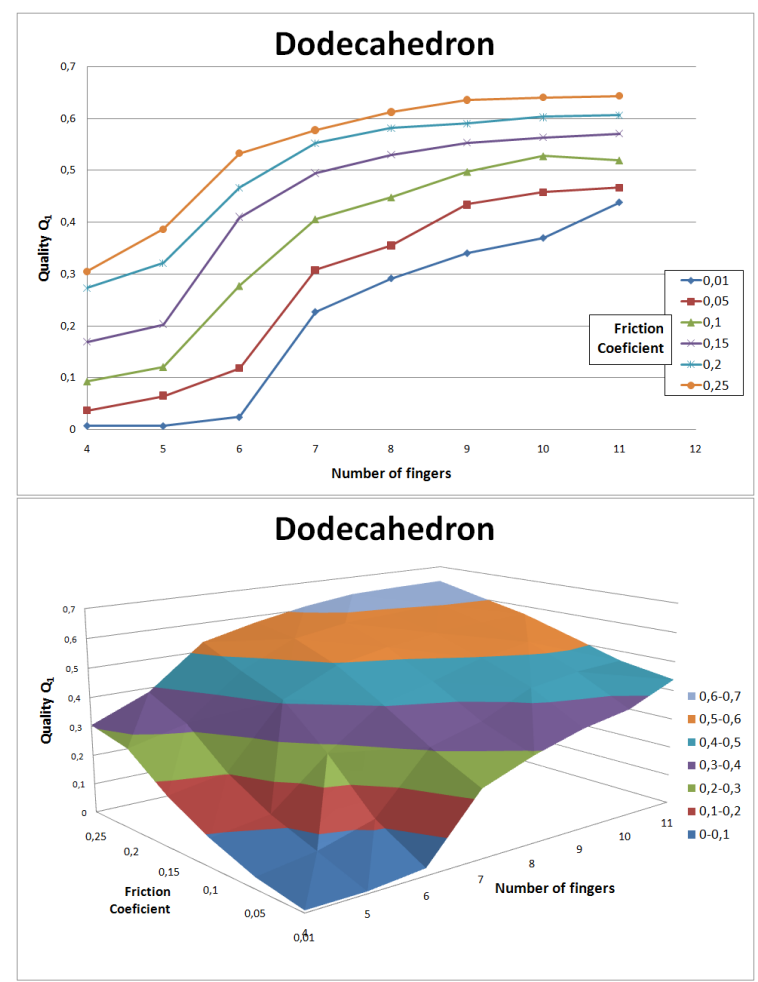

Fig. 10. Experiment results with the Dodecahedron.

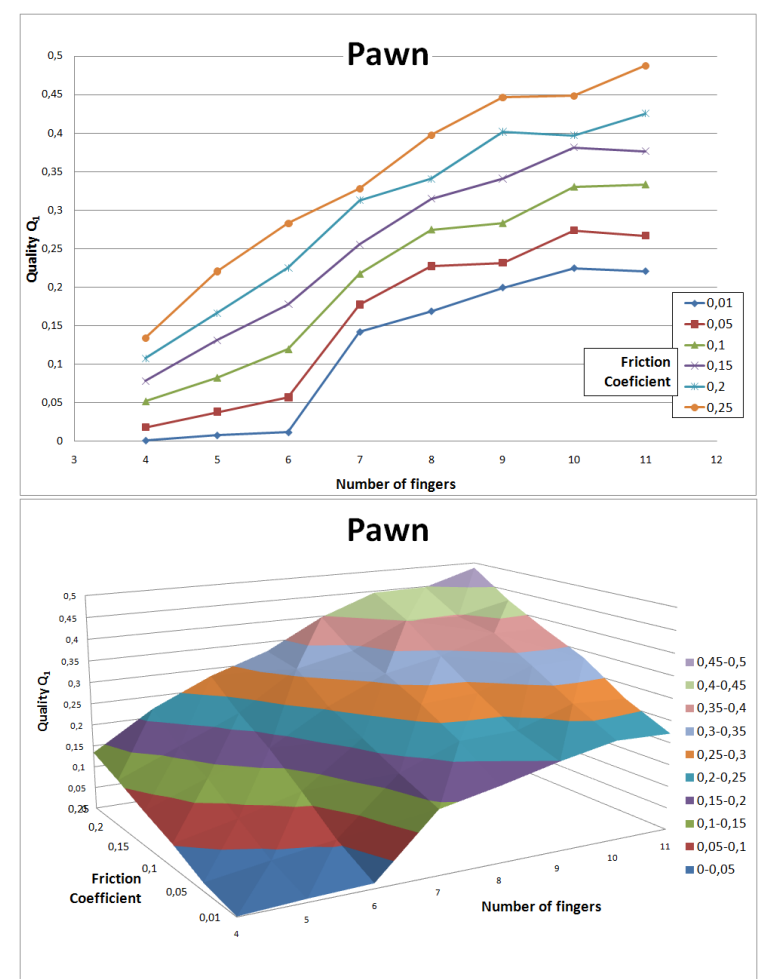

Fig. 11. Experiment results with the Pawn. to achieve a given desired minimum quality. A study over a wider variety of objects is now under development.

\section{REFERENCES}

[1] A. Bicchi, "On the closure properties of robotic grasping," Int. J. Robotics Research, vol. 14, no. 4, pp. 319-344, 1995.

[2] R. Suárez and J. Rosell, "Searching form-closure fixturing points on objects described by triangular meshes," in IEEE International Symposium Conference on Assemblyand Manufacturing, ISAM'2007, 2007, pp. 7-12.

[3] A. Bicchi and V. Kumar, "Robotic grasping and contact: A review," in Proc. IEEE ICRA 2000, 2000, pp. 348-352.

[4] Y. Liu, "Computing n-finger form-closure grasps on polygonal objects," Int. J. Robotics Research, vol. 19, no. 2, pp. 149-158, 2000.

[5] J. Cornellà and R. Suárez, "On computing form-closure grasps/fixtures for non-polygonal objects," in Proc. IEEE Int. Symp. Assembly and Task Planning, ISATP 2005, 2005, pp. 138-143.

[6] N. Niparnan and A. Sudsang, "Computing all force-closure grasps of 2D objects from contact point set," in Proc. IEEE/RSJ IROS 2006, 2006, pp. 1599-1604.

[7] J. Ponce, S. Sullivan, A. Sudsang, J. Boissonat, and J. Merlet, "On computing four-finger equilibrium and force-closure grasps of polyhedral objects," Int. J. Robotics Research, vol. 16, no. 1, pp. 1135, 1997.

[8] D. Ding, Y. Liu, and S. Wang, "Computation of 3-D form-closure grasps," IEEE Trans. Robotics and Automation, vol. 17, no. 4, pp. 515-522, 2001.

[9] X. Zhu and J. Wang, "Synthesis of force-closure grasps on 3-D objects based on the Q distance," IEEE Trans. Robotics and Automation, vol. 19, no. 4, pp. 669-679, 2003.

[10] X. Zhu and H. Ding, "Planning force-closure grasps on 3-D objects," in Proc. IEEE ICRA 2004, 2004, pp. 1258-1263.

[11] Y. Liu, M. Lam, and D. Ding, "A complete and efficient algorithm for searching 3-D form closure grasps in the discrete domain," IEEE Trans. Robotics, vol. 20, no. 5, pp. 805-816, 2004.

[12] N. Niparnan and A. Sudsang, "Fast computation of 4-fingered forceclosure grasps from surface points," in Proc. IEEE IROS 2004, 2004, pp. 3692-3697.

[13] M. Roa and R. Suárez, "Finding locally optimum force-closure grasps," Robotics and Computer-Integrated Manufacturing, vol. 25, no. 3, pp. 536-544, 2009.

[14] M. Alexa, J. Behr, D. Cohen, S. Fleishman, D. Levin, and C. Silva, "Computer and rendering point set surfaces," IEEE Trans. Visualization and Computer Graphics, vol. 9, no. 1, p. 315, 2003.

[15] R. Campbell and Flynn, "A survey of free-form object representation and recognition techniques, computer vision and image understanding," Computer Vision and Image Understanding, vol. 81, p. 166210, 2001.

[16] M. Roa, R. Suárez, and J. Cornellà, "Revisión de medidas de calidad para la prensión de un objeto," Revista Iberoamericana de Automtica e Informatica Industrial, vol. 5, no. 12, pp. 66-82, 2008.

[17] Z. Li and S. Sastry, "Task-oriented optimal grasping by multifingered robotic hands," in Proc. IEEE ICRA 1987, 1987, pp. 389-394.

[18] B. Kim, S. Oh, B. Yi, and I. Suh, "Optimal grasping based on nondimensionalized performance indices," in Proc. IEEE IROS 2001, 2001, pp. 949-956.

[19] B. Mirtich and J. Canny, "Easily computable optimum grasps in 2D and 3D," in Proc. IEEE ICRA 1994, 1994, pp. 739-747.

[20] Y. Maeda, K. Oda, and S. Makita, "Analysis of indeterminate contact forces in robotic grasping and contact tasks," in IEEE/RSJ Int. Conf. on Intelligent Robots and Systems, 2007, pp. $1570-1575$.

[21] C. Ferrari and J. Canny, "Planning optimal grasps," in Proc. IEEE ICRA 1992, 1992, pp. 2290-2295.

[22] D. Kirkpatrick, B. Mishra, and C. Yap, "Quantitative Steinitz's theorem with applications to multifingered grasping," Discrete and Computational Geometry, vol. 7, no. 3, pp. 295-318, 1992.

[23] A. Miller and P. Allen, "Examples of 3D grasp quality computations," in Proc. IEEE ICRA 1999, 1999, pp. 1240-1246.

[24] A. Miller and P. K. Allen, "Graspit!: A versatile simulator for robotic grasping," IEEE Robotics and Automation Magazine, vol. 11, no. 4. 